\title{
THE INVESTIGATION OF TIME-VARYING SYNCHRONY OF EEG DURING SENTENCE LEARNING USING WAVELET ANALYSIS
}

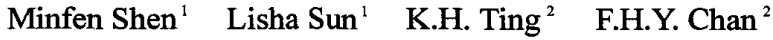 \\ 1. Science Research Centre, Shantou University, Guangdong 515063, China. \\ Email: mfshen@stu.edu.cn, \\ 2. Department of Electronic Engineering, The University of Hong Kong, Hong Kong, China.
}

\begin{abstract}
The synchrony analysis has been used as a tool for the purpose of investigating how the cognitive processes take place between different brain regions when the specified learning task is going on. We propose a novel method based on the time-frequency representation for quantifying synchronization between two channel EEG with both temporal and spectral resolution. The presented method employed the wavelet transform for cross coherent spectral analysis of the EEG signals recorded during sentences recognizing and learning. The wavelet-coherent magnitude spectra provide the information indicating the degree of coherence and the cross-wavelet phase relation serves to indicate the direction of information flow between two EEG channels on different cortical regions. Real EEG recordings are collected based on a cognitive target. It is observed from both the magnitude spectra and phase of the wavelet coherence that there are obvious differences between the identification of both Chinese and English sentences. These are helpful for the research on the English study for Chinese students.
\end{abstract}

\section{INTRODUCTION}

There have been a great deal of research results indicating that the brain works as a distributed parallel processing system. The study of neural synchronization of EEG signals can help us to understand the underlying cognitive processes. When some task of learning is going on, cognitive processes take place in different brain regions. Investigating neural synchronization can help us to understand the underlying cognitive processes. Conventional coherence analysis, as a linear method, employed the correlation for analysing the synchronization between two channel EEG signals. To measure the degree of synchronization the conventional coherence analysis is refereed a useful tool for the multi-channel EEGs to calculate the normalized correlation between two time series in the frequency domain. Some results have shown that the coherence of EEG recorded from different sites is usually closely correlated with cognitive processes [1-5] However, as the changes of the physiological states and the relative environment when the cognitive and information processing take place, the statistical properties of EEG usually change with time. Many practical EEG signals turn out to be extremely non-stationary processes. It is clear that the routine coherence analysis based on the assumption of stationarity fail to deal wit the time-varying EEG signals since most conventional cross-correlation methods lack the temporal resolution for the non-stationary EEG [6], which limits the dynamic synchrony analysis of practical EEG signals.

For this purpose, this paper proposes a modern time-frequency method, which provides time-varying coherent analysis via continuous wavelet transform. This novel method combines the wavelet transform with the traditional coherence analysis, which employs an alternative way for quantifying synchronization of the signals with both temporal and spectral resolution. Wavelet coherent spectrum is defined and computed from the EEG data set such that the cross wavelet magnitude spectra serves to indicate the degree of coherence and the cross wavelet phase can be used to provide the direction of information flow between two channel signals on different cortical regions. The wavelet coherent analysis can explore the amount of synchrony among multiple channels, and are used to investigate the synchronization and the 
corresponding information processing of the EEG signals recorded during identifying the specified sentences. The simulation and some real EEG data analysis under specified cognitive task are also demonstrated.

\section{CROSS-SPECTRUM AND COHERENCE}

In many applications to the analysis of the EEG, we need to study whether or not two signals are linearly related. Generally, cross spectral analysis and coherence have been widely used to investigate the linear relationship of frequency components in two EEG signals for the purpose of studying the cognitive processes between two different brain regions.For two different random process $x(t)$-and $y(t)$, investigate the linear coherent relationship between two signals, the correlation function is defined as

$$
R_{x y}(\tau)=E[x(t) y(t+\tau)]
$$

The cross-spectral density function of $x(t)$ and $y(t)$ can be defined as

$$
S_{x y}(f)=E\left[X(f) Y^{*}(f)\right]
$$

The cross-spectral density function has a magnitude relationship with the PSD of the processes $x(t)$ and $y(t)$ :

$$
S_{x}^{-}(f) S_{y}(f) \geq\left|S_{x y}(f)\right|^{2}
$$

The squared coherence function (SCF) is defined as:

$$
\gamma_{x y}^{2}(f)=\left|S_{x y}(f)\right|^{2} / S_{x}(f) S_{y}(f)
$$

Thus, the bound of SCF is between 0 and 1 such as

$$
0 \leq \gamma_{x y}^{2}(f) \leq 1
$$

The coherence can be expressed as a complex form from the SCF. The complex coherence function (CCF) is defined as

$$
\gamma_{x y}(f)=\sqrt{\gamma_{x y}^{2}(f) \angle S_{x y}(f)}
$$

Evidently, the CCF contains both amplitude and phase information between frequency components in the processes $x(t)$ and $y(t)$. The amplitude of CCF reflects the significant linear relationship between $x(t)$ and $y(t)$, and the phase of CCF reflects the time shift between $x(t)$ and $y(t)$.

\section{WAVELET COHERENCE}

The wavelet analysis is used as an efficient method for investigating the local characteristics of the non-stationary or time-varying signals. The wavelet transform is an alternative to other time frequency distribution with the advantage of being linear, producing a multiresolution decomposition of the signals. Thus, the wavelet transform provide us a way to deal with the non-stationary processes. To measure the degree of the time-varying synchronization of the underlying non-stationary EEG signals, cross wavelet spectral analysis is proposed due to the wavelet's sufficient temporal characteristics.

Developed with the wavelet analysis for non-stationary signal, cross wavelet analysis can be obtained to explore the amount of synchrony among the multiple channel signals. Among various mother wavelest, the Morlet wavelet, as a complex wavelet funcion, is chosen as we can benefits from its nature of complex properties, which provide both magnitude and phase information between two EEG channels. The continuous wavelet transform of the EEG time series is given by [7-8]

$$
W T_{x}(\tau, a)=|a|^{-1 / 2} \int_{-\infty}^{\infty} x(t) \varphi\left(\frac{t-\tau}{a}\right) d t
$$

$$
\varphi(t)=e^{-t^{2} / 2} e^{j w_{0} t}
$$

Based on the equation (2), the cross wavelet spectra can be defined as

$$
\begin{gathered}
C W S_{x y}(\tau, a)=W T_{x}(\tau, a) W T_{y}^{*}(\tau, a) \\
=\left|C W S_{x y}(\tau, a)\right| \phi(\tau, a)
\end{gathered}
$$

The cross wavelet magnitude spectra $\left|X_{i j}(\tau, a)\right|$ reflects the degree of coherence between two signals while the phase relationship $\phi_{i j}(\tau, a)$ reveals the direction of information flow between the two signals. Since phase 
difference at a particular frequency between two channels is meaningful only when the two channels are highly coherent at this frequency, a masking operation on the cross wavelet phase is desirable.

To test the behaviour of the proposed method, two simulated time series with different frequency is generated as

$$
X(t)=\sin 2 \pi f_{1}(t) t+\sin 2 \pi f_{2}(t) t,
$$

where $f_{1}(t)=t, f_{2}(t)=t+2$.

According to the definition of the wavelet coherence, the two time series should synchronize within all the time. The process is given in Fig. 1. (a). Through the associated local cross spectral analysis, we obtained the corresponding wavelet cross spectral representation of the non-stationary data. Fig.1.(b) shows the result. The results clearly indicate that wavelet coherent analysis gives a time and frequency relation with high resolution. The phase difference is also consistent to the wavelet coherence representations. The phase values that are out of our interest in this paper can therefore be ignore for better visualization.

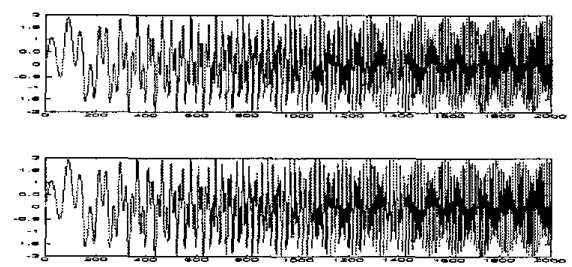

(a)

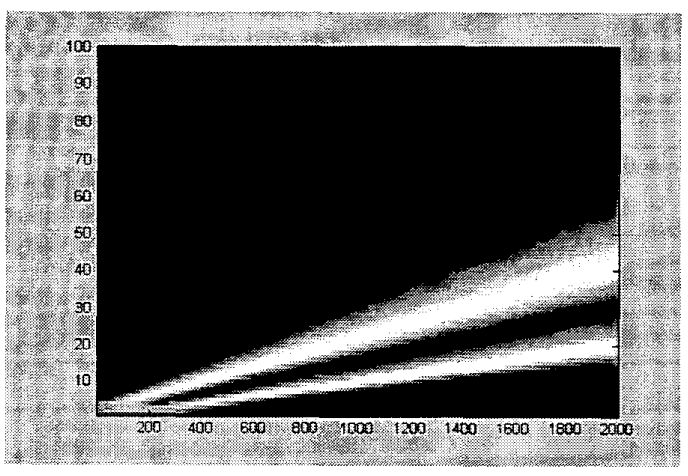

(b)

Figure 1. Simulation for wavelet coherent analysis. (a) 2000 points bivariate time series oscillate at different frequency at different time. (b) The wavelet-coherent spectrum from $1-100 \mathrm{~Hz}$.

\section{EEG ANALYSIS AND DISCUSSION}

EEGs were recorded with the 128-channel Geodesic EEG/ERP system with the sampling frequency of $250 \mathrm{~Hz}$. The EEG data were collected based on a cognitive target detection experiment to demonstrate the difference between the identification of Chinese and English sentence. Subjects were presented with visual stimulus of Chinese or English sentence. The sentence stimulus duration was $25 \mathrm{~ms}$, and then $26-250 \mathrm{~ms}$ was the identification duration. Subjects were requested to give a reaction (as press a button) when the grammar of the sentence was identified as true or false. Our interest was focused on the synchrony with the front-right regions of the brain.

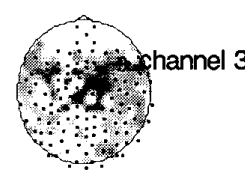

(a)

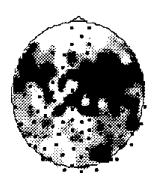

(b)

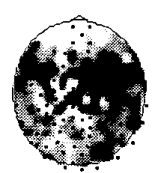

(c)
Fig.2. Wavelet-coherent spectra when subjects identified the Chinese sentences. (a) The average spectrum of the coherence during the first periods of $1-25 \mathrm{~ms}$. (b) The result during the second periods of $26-150 \mathrm{~ms}$. (c) The result during the final periods of $151-250 \mathrm{~ms}$.

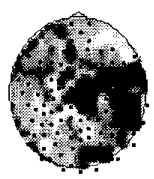

(d)

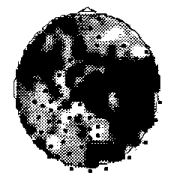

(e)

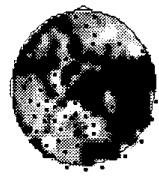

(f)
Fig.3. Wavelet-coherent spectra when subjects identified the English sentences with three same periods as in Fig.2.

The channel 3 was selected as a reference point. We developed a colour imaging method to describe the spatial representation of the coherences in each pair of EEGs induced by a cognitive task. Fig. 2 and Fig. 3 show the wavelet-coherent spectra within the frequency from 18 to 
$25 \mathrm{~Hz}$ between channel 3 and other 127 channels. The coherent maps are based on the correlation of the electrical activities of the areas on the scalp. The time-varying coherent distributions were demonstrated from Fig.2 (a) to (c) and from Fig.3 (a) to (c). Fig.2 represents the results during identifying the Chinese sentence while Fig. 3 did the English sentence.

Based on the preliminary analysis, there exists a significant difference between first period $(1-25 \mathrm{~ms})$ and the second period $(26-150 \mathrm{~ms})$ for processing the identification of both English and Chinese sentences. A lot of information exchanges take place during the period of "sentence justification". Also it can be seen that the region of information exchange when performing the Chinese sentence identification is on the middle of the brain, whereas the significant region of information exchange during English sentence justification is on the heel of the brain.

\section{CONCLUSION}

In this contribution, a time-frequency coherence analysis, wavelet coherence, of EEG signals is proposed for investigating the time-varying synchrony between the different brain regions in a cognitive task of identifying sentences in both Chinese and English. Wavelet coherence is defined which enables us to investigate the synchronization and the information flow during processing the cognitive task of justifying sentences in Chinese and English via both the wavelet coherent spectrum and the cross wavelet phase relationship. Morlet mother wavelet is first applied on the raw EEG multichannel signals. Cross wavelet spectra is defined such that the cross magnitude spectra reveals the localized coherency power in the time-frequency plane and the cross phase spectra reveals the direction of information flow from one channel to another. For Chinese students, the different regions of the brain have different degree of synchrony and information exchange patterns during processing the identification of Chinese and English sentence.

\section{ACKNOWLEDGEMENT}

This work is supported by the Key Grant of Natural Science Foundation of Guangdong 2002.
[1] P. L. Nunez and R. Srinivasan, "EEG coherence I: statistics, reference electrode, vdume conduction, 1 aplacians. cortical imaging and interpretation at multiple scales". Elect. and Clin Neurophys. Vol.103, 1995, pp.499-515

[2] C. W. Anderson, E. A. Stolz, "Multivariate autoregressive models for classification of spontaneous electroencephalogram during mental tasks". IEEE Trans Biomedical Eng. Vol.45(3), 1998, pp.277-86.

[3] Miltner WHR, Braun C, Arnold M, Witte H, Taub E. Coherence of gamma-band EEG activity as a basis for associative learning. Nature 1999;397:434-6.

[4] S. Weiss and P. Rappelsberger, "EEG coherence within $13-18 \mathrm{~Hz}$ band as a correlate of a distinct lexical organization of concrete and abstract nouns in humans", Neuroscience Letters, vol.209, 1996, pp.17-20.

[5] Gazzaniga MS, Ivry RB, Mangun GR. Cognitive neuroscience: the biology of the mind. NY: W.W.Norton, 1998.

[6] E. Moller and B. Schack, "Instantaneous multivariate EEG coherence analysis by means of adaptive high-dimensional auto regressive models", J. of Neuroscience Methods. Vol.105; 2001, pp.143-158.

[7] Meyer Y. Wavelets and Operators. Cambridge: Cambridge University Press, 1992.

[8] Daubechies I. Ten lectures on wavelets. Philadelphia: SIAM, 1992.

\section{REFERENCES}

